\title{
Network Pharmacology and Experimental Validation to Reveal the Pharmacological Mechanisms of Liuwei Dihuang Decoction Against Intervertebral Disc Degeneration
}

Huihao Zhang, ${ }^{1}, *$ Sai Yao, ${ }^{1} *$ Zhiguo Zhang, '** Chengcong Zhou, Fangda Fu, 'Yishan Bian,' Huan Luo,' Yan Li,' Shuxin Yan,' Yuying Ge, (D) Yuying Chen, ${ }^{3}$ Kunyu Zhan, ${ }^{4}$ Yanzhi Ge,' Zuxiang Chen, $\mathbb{D D}^{1}$ Ming Yue, ${ }^{5}$ Xiaofeng Li, ${ }^{6}$ Weibin Du, (1) 1,7 Hongting Jin, Peijian Tong,' Hongfeng Ruan, ${ }^{1,8}$ Chengliang $\mathrm{Wu}^{\prime}$

'Institute of Orthopaedics and Traumatology, The First Affliated Hospital of Zhejiang Chinese Medical University, Hangzhou, Zhejiang, People's Republic of China; ${ }^{2}$ Department of Pharmacy, The Second Affiliated Hospital, School of Medicine, Zhejiang University, Hangzhou, Zhejiang, People's Republic of China; ${ }^{3}$ The Fourth Clinical Medical College, Zhejiang Chinese Medical University,

Hangzhou, Zhejiang, People's Republic of China

${ }^{4}$ The Second Clinical Medical College, Zhejiang Chinese Medical University, Hangzhou, Zhejiang, People's Republic of China; ${ }^{5}$ Department of Physiology, Zhejiang Chinese Medical University, Hangzhou, Zhejiang, People's Republic of China; ${ }^{6}$ Department of Orthopedics and Traumatology, Shanghai Municipal Hospital of Traditional Chinese Medicine, Shanghai University of Traditional

Chinese Medicine, Shanghai, People's Republic of China; ${ }^{7}$ Research Institute of Orthopedics, The Affliated JiangNan Hospital of Zhejiang Chinese Medical University, Hangzhou, Zhejiang, People's Republic of China; ${ }^{8}$ Longhua Hospital Affiliated to Shanghai University of Traditional Chinese

Medicine, Shanghai, People's Republic of China

*These authors contributed equally to this work

Correspondence: Hongfeng Ruan; Peijian Tong

Institute of Orthopaedics and

Traumatology, The First Affiliated

Hospital of Zhejiang Chinese Medical

University, Hangzhou, Zhejiang, 310053 ,

People's Republic of China

Email rhf@zcmu.edu.cn;

peijiantongzjtcm@।63.com
Purpose: To explore the pharmacological mechanisms of Liuwei Dihuang Decoction (LWDHD) against intervertebral disc (IVD) degeneration (IVDD) via network pharmacology analysis combined with experimental validation.

Methods: First, active ingredients and related targets of LWDHD, as well as related genes of IVDD, were collected from public databases. The protein-protein interaction (PPI) network, Gene Ontology (GO) and Kyoto Encyclopedia of Genes and Genomes (KEGG) functional enrichment analyses were performed to predict the core targets and pathways of LWDHD against IVDD. Secondly, the IVDD model of mice treated with LWDHD was selected to validate the major targets predicted by network pharmacology.

Results: By searching the intersection of the active ingredient targets and IVDD targets, a total of 110 targets matched the related targets of 30 active ingredients in LWDHD and IVDD were retrieved. PPI network analysis indicated that 17 targets, including Caspase-3, IL-1 $\beta$, P53, etc., were hub targets. GO and KEGG enrichment analyses showed that the apoptosis pathway was enriched by multiple targets and served as the target for in vivo experimental study validation. The results of animal experiments revealed that LWDHD administration not only restored the decrease in disc height and abnormal degradation of matrix metabolism in IVDD mice but also reversed the high expression of Bax, Caspase-3, IL-1 $\beta$, P53, and low expression of Bcl-2, thereby inhibiting the apoptosis of IVD tissue and ameliorating the progression of IVDD.

Conclusion: Using a comprehensive network pharmacology approach, our findings predicted the active ingredients and potential targets of LWDHD intervention for IVDD, and some major target proteins involved in the predictive signaling pathway were validated experimentally, which gave us a new understanding of the pharmacological mechanism of LWDHD in treating IVDD at the comprehensive level.

Keywords: intervertebral disc degeneration, Liuwei Dihuang decoction, network pharmacology, pharmacological mechanisms, apoptosis

\section{Introduction}

Low back pain (LBP), a common clinical disease worldwide, seriously lowers people's quality of life and happiness index and results in an enormous socioeconomic burden. ${ }^{1,2}$ Intervertebral disc degeneration (IVDD) has been widely recognized as a major cause of LBP and the leading cause of disability; however, the molecular mechanisms of LBP have not yet been fully elucidated. ${ }^{3-5}$ Recent studies indicated that exaggerated expressions of proinflammatory factors such as 
interleukin-1 $\beta$ (IL-1 $\beta$ ), as well as apoptosis, were positively correlated with the progression of IVDD. ${ }^{6,7}$ Current clinical treatments for IVDD include conservative treatment and surgical treatment, but they cannot restore or even reverse the pathological changes of IVDD and are often limited by side effects. ${ }^{8}$ Traditional Chinese medicine (TCM) herb formulae have the characteristics of "multi-component, multichannel", which have their own advantages and characteristics in personalized treatment and early intervention.

Liuwei Dihuang Decoction (LWDHD) is a classic prescription for TCM, and clinical practice for thousands of years has proved that it has very good effects on many kidney deficiency-induced diseases, including LBP., ${ }^{9,10}$ LWDHD, derived from the "Jingyue Quanshu", is composed of Rehmanniae Radix Praeparata (Shu Di Huang), Rhizoma Dioscoreae (Shan Yao), Cornus Officinalis (Shan Zhu Yu), Cortex Moutan (Mu Dan Pi), Alisma Orientale (Ze Xie), and Poria Cocos (Fu Ling). These six herbs act synergistically in a variety of ways to help the body regain its balance and homeostasis. Although some modern pharmacological experiments have demonstrated that LWDHD had anti-inflammatory, anti-oxidative, anti-apoptotic and anti-aging effects, ${ }^{11,12}$ the underlying comprehensive pharmacological mechanism of LWDHD on LBP or IVDD remains obscure.

Due to numerous bioactive components and complex chemical systems of herbal formulae, it is a challenge to decipher pharmacological mechanisms with routine pharmacologic analysis methods. ${ }^{13}$ With the development of computer technology and theory of system biology theory, network pharmacology is an emerging interdisciplinary subject, which has great advantages in interpreting the pharmacologic mechanisms of TCM with multiple components, targets and pathways, and is extremely commensurate with the holistic philosophy of TCM. ${ }^{14-16}$ By network pharmacological analysis, Liu et al found 28 compounds and 421 targets in Huaihua-San formed the main pathways linked to ulcerative colitis, which revealed the mechanism of Huaihua-San against ulcerative colitis. ${ }^{17}$ Similarly, with the help of network pharmacology, we predicted and verified the potential pharmacological mechanism of Bu-Yang herbs for postmenopausal osteoporosis via regulating the Wnt/ $\beta$-catenin signaling pathway. ${ }^{18}$ Therefore, all this evidence indicated that network pharmacology analysis may be a good tool for exploring the chemical compositions of LWDHD and its relationships with IVDD.
In this study, the active ingredients of LWDHD were firstly screened out based on the screening criteria (oral bioavailability (OB) $\geq 30 \%$ and drug-likeness (DL) $\geq 0.18$ ), and the potential targets of these active ingredients against IVDD were identified by the PPI network. Then, the GO and KEGG functional enrichment analyses were performed to explore the potential signaling pathways of LWDHD against IVDD. Finally, the major target proteins involved in the predicted pathway were further validated in the IVDD model of mice treated with LWDHD. A flowchart of this research is shown in Figure 1.

\section{Materials and Methods}

\section{Screening for Active Ingredients in LWDHD and Prediction of Their Targets}

The active ingredients in LWDHD were screened from the Traditional Chinese Medicine Systems Pharmacology (TCMSP) Database and Analysis Platform (http:// tcmspw.com/tcmsp.php $)^{19}$ by using "Shu Di Huang", "Shan Yao", "Shan Zhu Yu", "Mu Dan Pi", "Ze Xie" and "Fu Ling" as keywords to identify targets related to LWDHD. And $\mathrm{OB} \geq 30 \%$ and $\mathrm{DL} \geq 0.18$ were chosen as the screening criteria. ${ }^{20}$ All target names were standardized through the UniProt database (https://sparql.uni prot.org/). ${ }^{21}$

\section{Identification of Potential Targets for IVDD}

The potential targets of IVDD were retrieved from Genecards (https://www.genecards.org/), ${ }^{22}$ Online Mendelian Inheritance in Man (OMIM, https://www.genecards.org/), ${ }^{23}$ DrugBank (https://go.drugbank.com/) ${ }^{24}$ and PharmGkb (https://www. pharmgkb.org/). ${ }^{25}$ After eliminating duplicates, the potential targets for IVDD were identified.

\section{Construction of Ingredient-Target- Disease Network}

The common targets of active ingredients in LWDHD and potential targets for IVDD were collected and inputted into Cytoscape 3.8 software to construct an ingredients-targetdisease interaction network, which visualized and integrated topological parameters. And the importance of ingredients and targets was determined by degree, which indicated the total number of routes related to the node by other nodes. And the higher the degree value, the more important it was. 


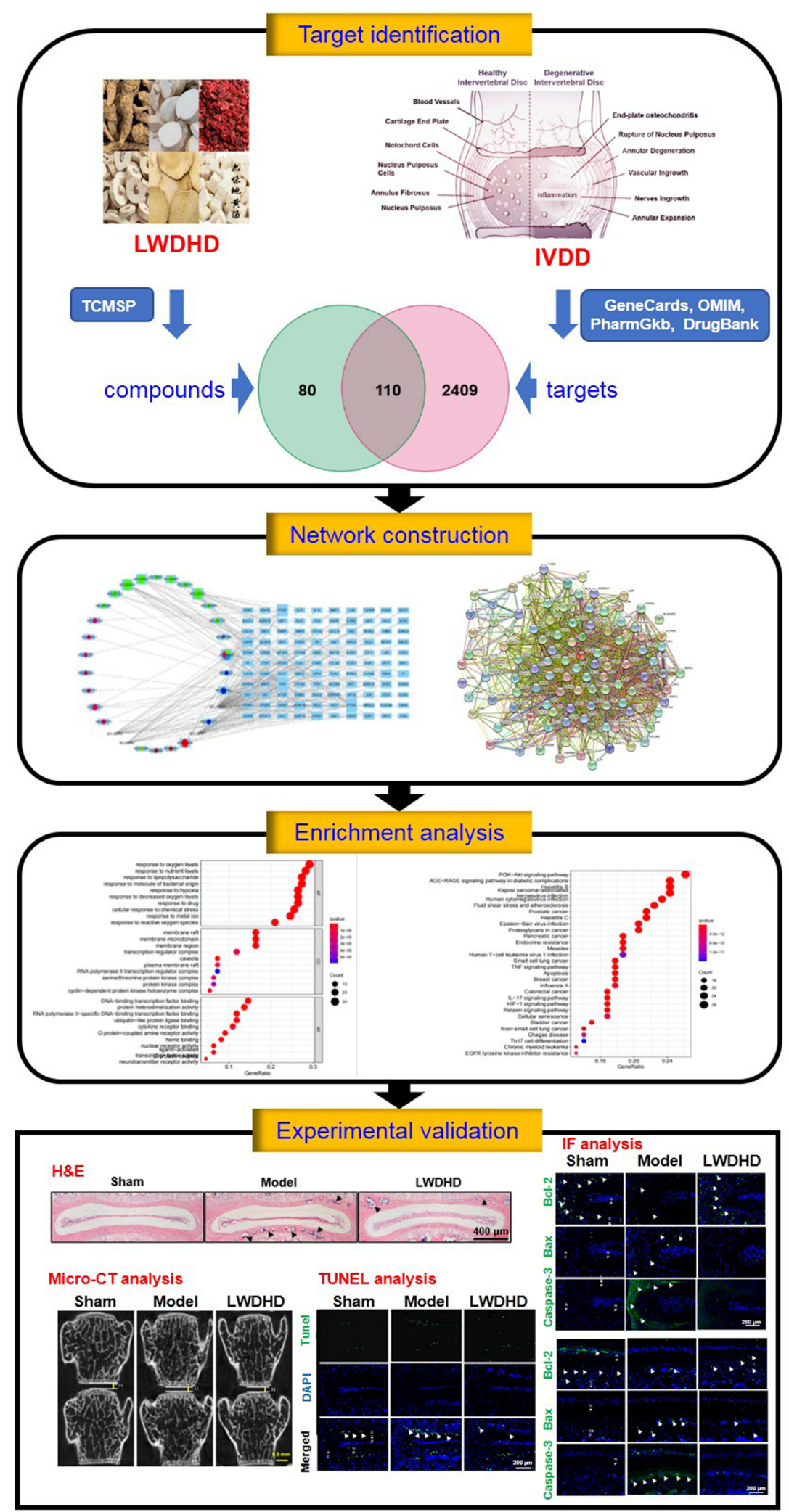

Figure I Integrated flowchart for illustrating the mechanism of LWDHD in the treatment of IVDD includes potential targets identification, PPI network construction, enrichment analysis, and experimental validation. 


\section{Construction of Protein-Protein Interaction (PPI) Network}

The String database built the functional connections of a protein network by collecting and integrating all known and predicted protein functional associations. ${ }^{26}$ The identified potential proteins were imported to String (https:// string-db.org/) to obtain corresponding relationships about protein interactions, and the PPI network was constructed via Cytoscape 3.8 software. Then, we used CytoNCA software to identify core proteins based on betweenness centrality (BC), closeness centrality (CC), degree centrality (DC), Eigenvector centrality (EC), local average connectivity-based method (LAC), and network centrality (NC). And BC, CC, DC, EC, LAC, NC should be greater than or equal to the median value. ${ }^{14}$

\section{Functional Enrichment Analyses}

$\mathrm{R}$ software, a free software environment for statistical computing and graphics, was used to perform GO and KEGG functional enrichment analyses for the key targets. The GO and KEGG pathway analyses were screened for $P<0.05$. The top 10 items of GO analysis and the top 30 items of KEGG analysis identified from R software were mapped as bar plots and bubble plots. Finally, a diagram of signaling pathways predicted by the KEGG analysis and associated with IVDD was generated by R software. ${ }^{27}$

\section{Chemicals and Reagents}

Primary antibodies against Aggrecan, Adamts5 and Mmp13 were purchased from Abcam Company Ltd. (Cambridge, MA, USA), and primary antibodies against Bax, Bcl-2, Collagen II (Col2) and P53 were ordered from Ruiying Biological Co. (Jiangsu, China). Primary antibody against Caspase-3 was from Hua'an Biological Co. (Hangzhou, China), and primary antibody against IL-1 $\beta$ was obtained from Bioss Inc. (Boston, MA, USA). Alexa Fluor ${ }^{\circledR} 488$ rabbit anti-goat $\mathrm{IgG}(\mathrm{H}+\mathrm{L})$ secondary antibody (green) was supplied by Zhongshan Jinqiao Biotech Co. (Beijing, China). TUNEL Bright Green Apoptosis Detection Kit was provided by Vazyme Biotech Co. (Nanjing, China). The remaining chemicals were obtained from Sigma-Aldrich (St. Louis, Mo, USA) unless otherwise stated.

\section{Preparation of LWDHD}

Six raw herbs of LWDHD were provided from the pharmacy department of the first affiliated hospital of Zhejiang Chinese Medical University (Hangzhou, China). Rehmanniae Radix Praeparata, Rhizoma Dioscoreae, Cornus Officinalis, Cortex Moutan, Alisma Orientale, and Poria Cocos were mixed as a proportion of 1.5:1.2:1.2:1:1:1 $(\mathrm{w} / \mathrm{w})$. The mixed herbs were immersed in 8 volumes of distilled water $(\mathrm{v} / \mathrm{m})$ for $1 \mathrm{~h}$, decocted for 2 times and $1.5 \mathrm{~h}$ each time. Then, the water-based decoction was concentrated to $0.6 \mathrm{~g} / \mathrm{mL}$ and stored at $-20^{\circ} \mathrm{C}$ before use. A voucher of specimen has been deposited at the First Affiliated Hospital of Zhejiang Chinese Medical University (Hangzhou, China) (Table 1).

\section{Animals and Experimental Grouping}

A total of $24 \mathrm{C} 57 \mathrm{BL} / 6 \mathrm{~J}$ mice (8-week-old, $22 \pm 2 \mathrm{~g}$ ) were supplied by the animal center of Zhejiang Chinese Medical University (Grade SPF, SCXK (Shanghai): 2017-0005). All of these mice were kept in a specific pathogen-free animal care facility and housed in an environmentally controlled room $\left(23 \pm 2^{\circ} \mathrm{C}\right.$, humidity $50 \pm 10 \%, 12 \mathrm{~h} / 12$ $\mathrm{h}$ light/dark cycle) and had free access to water and lab chow. All proposals concerning animals were approved by the Ethics Committee of Zhejiang Chinese Medical University (2020-KL-159-02), and all laboratory procedures were performed following the Regulations for the Administration of Affairs Concerning Experimental Animals approved by the State Council of the People's Republic of China.

Table I Detailed Information of Liuwei Dihuang Decoction (LWDHD)

\begin{tabular}{|l|l|c|c|c|}
\hline Chinese Name & Latin Name & Parts Used & Place of Origin & Voucher Specimen No. \\
\hline Shu Di Huang & Rehmanniae Radix Praeparata & Root & Hubei, China & 151,301 \\
Shan Yao & Rhizoma Dioscoreae & Root & Henan, China & 151,302 \\
Shan Zhu Yu & Cornus Officinalis & Fruit & Shanxi, China & 151,303 \\
Mu Dan Pi & Cortex Moutan & Root & Anhui, China & 151,304 \\
Ze Xie & Alisma Orientale & Stem & Jilin, China & 151,305 \\
Fu Ling & Poria Cocos & Sclerotium & 151,306 \\
\hline
\end{tabular}


All the mice were randomly and equally divided into 3 groups: Sham group, LSI group, LWDHD group $(\mathrm{n}=8$ in each group).

\section{IVDD Modeling and Drug Administration}

IVDD model was established by lumbar spine instability (LSI)-surgery as we previously reported. ${ }^{28}$ Briefly, the mice were anesthetized with $1.5 \%$ isoflurane (R510-2216, RWD Life Science, Shenzhen, China) in 100\% oxygen flowing at $1.0 \mathrm{~L} / \mathrm{min}$ and placed on the surgical table in a prone position. Then, a $2 \mathrm{~cm}$ longitudinal incision was made along the midline of the back to expose the lumbar 3 rd-5th spinous processes. The spinous processes along with supraspinous and interspinous ligaments were resected. Finally, the muscles and skin were sutured successively to allow the mice to recover. Mice in the LSI and LWDHD groups received the IVDD modeling, while mice in the Sham group received a similar surgical approach without manipulating spinous processes.

Three days after LSI surgery, mice in the LWDHD group were orally administered with LWDHD $(0.2 \mathrm{~mL} / 10$ $\mathrm{g}$ body weight) once a day for 8 consecutive weeks. Mice in the other 2 groups were given the same dosage of normal saline. Eight weeks after the LSI surgery, all mice were euthanized, and the lumbar vertebrae were collected for further analysis.

\section{Micro-CT Analysis}

Before histological processing, the vertebrae fixed with paraformaldehyde were analyzed using micro-CT equipment (Skyscan 1176, Bruker micro-CT N.V., Kontich, Belgium). And the vertebrae were scanned at a voltage of $50 \mathrm{kV}$ with a current of $500 \mu \mathrm{A}$ and a resolution of $9 \mu \mathrm{m}$ per pixel. The three-dimensional (3D) reconstruction of the spine was realized by NRecon v1.6 and CTAn v1.15 software. ${ }^{29}$

\section{Histological, Immunohistochemistry (IHC) and Immunofluorescence (IF) Analyses}

After fixation in $4 \%$ paraformaldehyde for 3 days, the tissues were decalcified with $14 \%$ EDTA solution $(\mathrm{pH}=7.4)$ for 21 days, dehydrated and embedded in paraffin. The lumbar tissues were processed into 5 - $\mu \mathrm{m}$-thick coronal-oriented sections and stained with hematoxylin-eosin (H\&E) ${ }^{28}$ For IF assay, sections were incubated with primary antibodies of Aggrecan (diluted 1:300), Col-II (diluted 1:500), Mmp3 (diluted 1:300), Adamts5 (diluted 1:300), Bcl-2 (diluted 1:300), Bax (diluted 1:300), Caspase-3 (diluted 1:300), P53 (diluted 1:500), IL-1 $\beta$ (diluted $1: 500$ ) at $4^{\circ} \mathrm{C}$ overnight. For IHC analysis, secondary biotinylated goat anti-rabbit antibody (diluted 1:1000) (Invitrogen) was added for $30 \mathrm{~min}$ the following day. A diaminobenzidine solution (Invitrogen) was used to detect IHC staining, followed by counterstaining with hematoxylin. For IF analysis, secondary goat anti-rabbit antibody conjugated with fluorescence (diluted 1:1000) was added for $30 \mathrm{~min}$ the next day. The number of positive cells was calculated in a blinded manner with Image-Pro Plus 6.0 (Media Cybernetics, Silver Spring, MD, USA) as we previously described. ${ }^{29}$

\section{TUNEL Assay}

The TUNEL Bright Green Apoptosis Detection Kit (Vazyme Biotech, Nanjing, China) was used to detect apoptosis of IVD cells as we previously described. ${ }^{29}$ All operations were performed in accordance with the manufacturer's instructions. Using Image-Pro Plus 6.0 (Media Cybernetics, Silver Spring, MD, USA), the number of positive cells was quantified by using three sections from each sample in three randomly selected fields of view. The total number of cells was estimated by DAPI staining.

\section{Statistical Analysis}

All numerical data were presented as median (min-max). SPSS statistical software package (SPSS 19.0 version; SPSS Inc., Chicago, Illinois, USA) was used for statistical analysis. Significant differences were analyzed by non-parametric Kruskal-Wallis test followed by Dunn's multiple comparisons test. $P<0.05$ was considered statistically significant.

\section{Results}

\section{Active Ingredients in LWDHD and Target Prediction}

By retrieving the TCMSP database and meeting the screening criteria ( $\mathrm{OB} \geq 30 \%$ and $\mathrm{DL} \geq 0.18$ ), a total of 74 active ingredients of LWDHD were selected, including 2 from Shu Di Huang, 20 from Shan Zhu Yu, 16 from Shan Yao, 11 from $\mathrm{Mu}$ Dan Pi, 15 from $\mathrm{Fu}$ Ling, and 10 from $\mathrm{Ze}$ Xie. Subsequently, a total of 584 potential targets of LWDHD were retrieved from the TCMSP database, containing 34 types in Shu Di Huang, 130 types in Shan Zhu Yu, 144 types in Shan Yao, 237 types in Mu Dan Pi, 30 types in Fu Ling, and 9 types in Ze Xie. After filtering out duplicate targets, a total of 190 targets were preserved for further research. 


\section{Potential Targets Prediction of IVDD}

By searching for research reports related to IVDD in 4 databases, 770 targets of IVDD were obtained from the GeneCards database, 118 targets of IVDD were obtained from the OMIM database, 1948 targets of IVDD were retrieved from the PharmGkb database, 9 targets of IVDD were obtained from the DrugBank database. After expurgating duplicates, a total of 2519 targets were stored, which were closely related to the occurrence and development of IVDD.

\section{Ingredient-Target-Disease Network}

To identify the intersection of LWDHD target genes and IVDD target genes, the Venn diagram analysis was carried out. As shown in Figure 2A, a total of 110 target genes were identified, which matched with the related targets of 30 active ingredients. Then, to elucidate the relationship between active ingredients and potential targets as well as IVDD, an ingredient-target-disease network was constructed by Cytoscape 3.8 software, consisting of 140 nodes and 250 edges (Figure 2B). The top 10 key active ingredients of LWDHD in the treatment of IVDD are enumerated in Table 2.

\section{PPI Network Construction and Key Targets}

To identify the core proteins of LWDHD intervention for IVDD, a PPI network with 109 nodes and 1753 edges was constructed using String database after removal of one target that was not linked to the other targets (Figure 3A). Then, we used the CytoNCA to calculate topological parameters, and screen the core proteins in two steps based on the

A

B
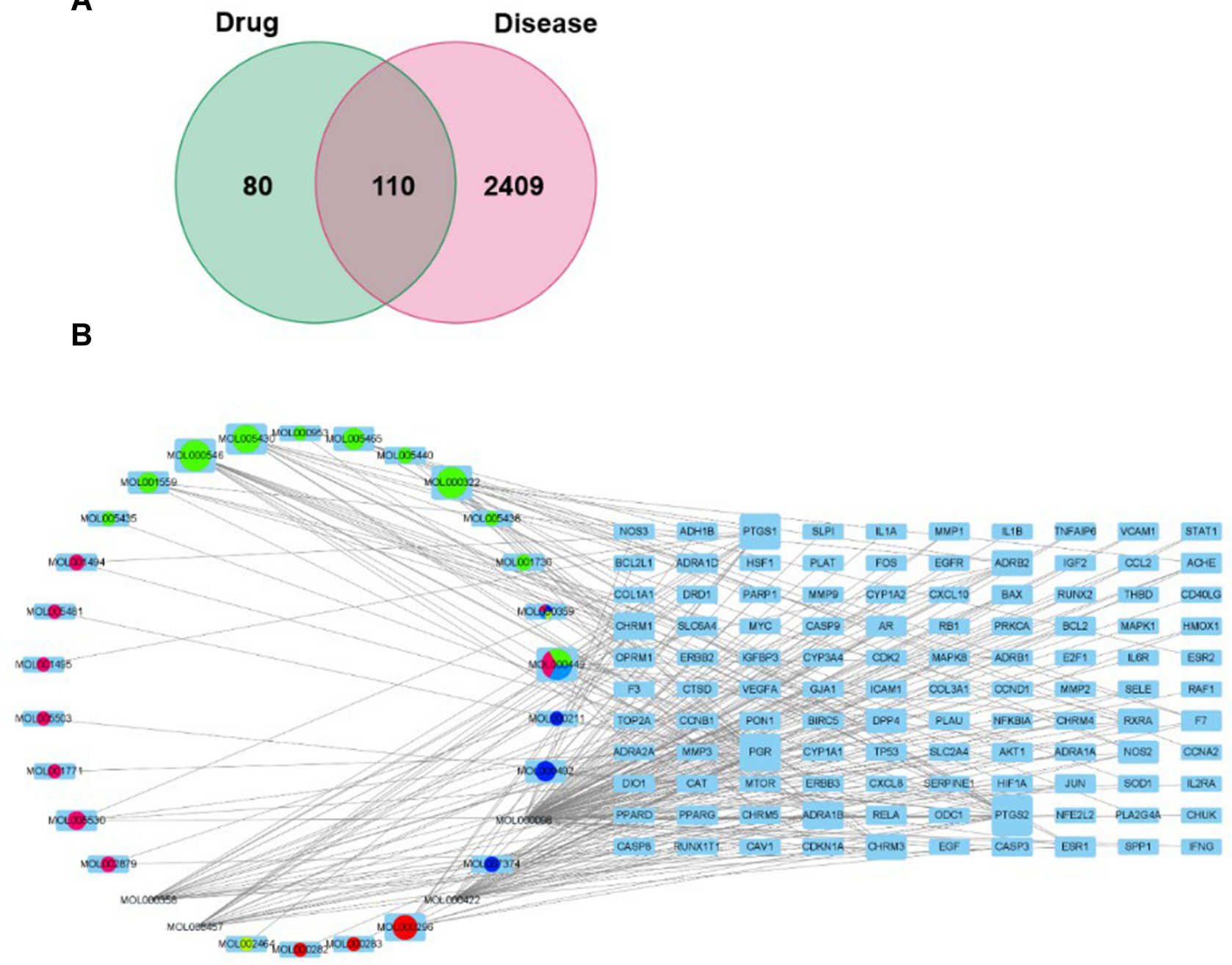

Figure 2 Construction of ingredient-target-disease network. (A) Venn diagram summarizing the intersection targets of the LWDHD and IVDD. (B) The network of Ingredient-target-disease. There were 6 kinds of herbs, 30 active components, 110 target genes. In this network, the ring on the left represented the composition and the rectangle on the right represented target genes. 
Table 2 The Top 10 Key Active Ingredients of Liuwei Dihuang Decoction in the Treatment of IVDD

\begin{tabular}{|c|c|c|c|c|c|}
\hline Molecular ID & Ingredient & Degree & Source & OB(\%) & DL \\
\hline MOL000098 & Quercetin & 86 & Mu Dan Pi & 46.43 & 0.28 \\
\hline MOL000422 & Kaempferol & 31 & Mu Dan Pi & 41.88 & 0.24 \\
\hline MOL000358 & Beta-sitosterol & 20 & Shan Zhu Yu & 36.91 & 0.75 \\
\hline MOL008457 & Tetrahydroalstonine & 17 & Shan Zhu Yu & 32.42 & 0.81 \\
\hline MOL000322 & Kadsurenone & 12 & Shan Yao & 54.72 & 0.38 \\
\hline MOL000449 & Stigmasterol & 12 & $\begin{array}{l}\text { Shu Di Huang, } \\
\text { Shan Yao, } \\
\text { Shan Zhu Yu }\end{array}$ & 43.83 & 0.76 \\
\hline MOL000546 & Diosgenin & 12 & Shan Yao & 80.88 & 0.81 \\
\hline MOL005430 & Hancinone $\mathrm{C}$ & 10 & Shan Yao & 59.05 & 0.39 \\
\hline MOL000296 & Hederagenin & 8 & Fu Ling & 36.91 & 0.75 \\
\hline MOL005465 & AIDSI80907 & 6 & Shan Yao & 45.33 & 0.77 \\
\hline
\end{tabular}

thresholds of $\mathrm{BC}, \mathrm{CC}, \mathrm{DC}, \mathrm{EC}, \mathrm{LAC}$, and NC. In the first step, BC, CC, DC, EC, LAC and NC should be greater than or equal to $30.37169029,0.5625,28,0.080398135$, 21.26666667 and 22.95852281 , respectively. In the second step, BC, CC, DC, EC, LAC and NC should be greater than or equal to $5.520157823,0.8666666667,33,0.157315858$, 28.24242424 and 30.735145735, respectively (Figure 3B). Consequently, a total of 17 core proteins were identified, including Caspase-3, IL-1 $\beta$, P53, CCL2, MAPK8, JUN, PTGS2, AKT1, MMP9, CXCL8, EGFR, EGF, MMP2, MAPK1, VEGFA, MYC, FOS, which were closely related to IVDD (Figure 3C).

\section{Functional Enrichment Analysis}

Next, to investigate the potential signaling pathways or biological processes regulated by LWDHD, the GO and KEGG functional enrichment analyses were carried out based on the potential targets of LWDHD. The results of GO analysis showed that the 110 genes were enriched in $2321 \mathrm{GO}$ entries $(P<0.05)$, including 2111 biological progress (BP), 59 cellular components (CC) and 151 molecular functions (MF), and the top 10 entries of BP, CC and MF are shown in Figure 4A. BP analysis showed that potential targets were primarily focused on the response to oxygen levels, response to lipopolysaccharide, response to molecule of bacterial origin, etc., while $\mathrm{CC}$ analysis showed that potential targets were primarily focused on membrane raft, membrane microdomain, membrane region, etc. In addition, MF analysis showed that potential targets were primarily focused on $\mathrm{G}$ proteincoupled amine receptor activity, DNA-binding transcription factor binding, protein heterodimerization activity, etc.

To analyze the representative pathways related to the core targets, the KEGG enrichment analysis was carried out, and the corresponding results revealed that a total of 163 significantly enriched signaling pathways were obtained $(P<0.05)$. The 30 most significant KEGG

A

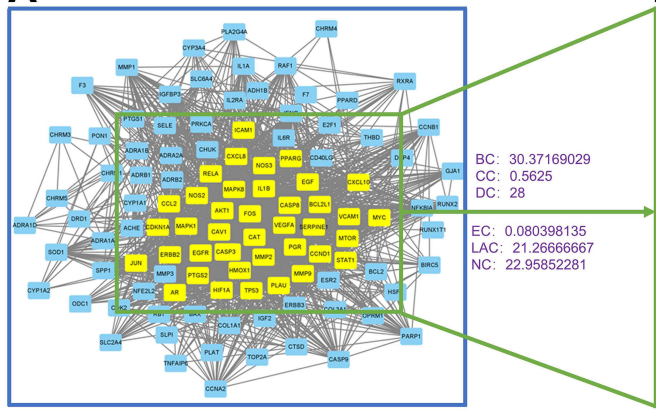

109 Nodes, 1753 Edges
B

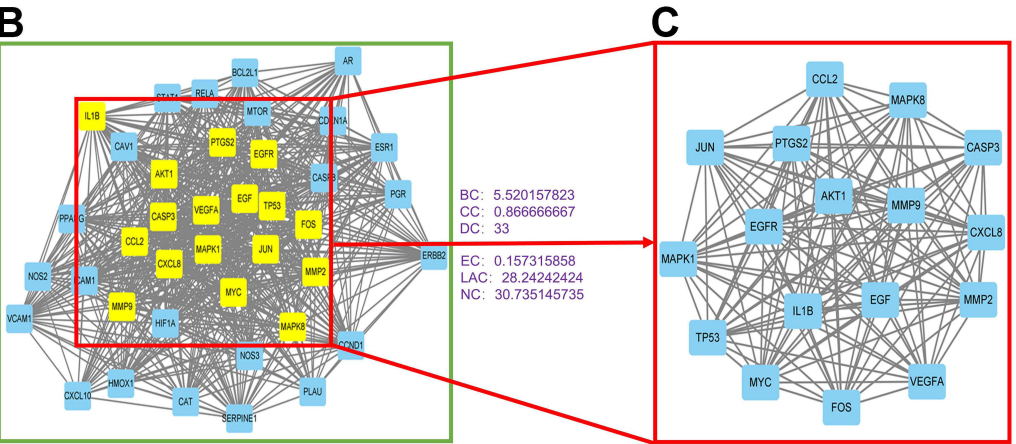

40 Nodes, 659 Edges

17 Nodes, 136 Edges

Figure 3 PPI network and cluster analysis of the disease targets. (A) PPI network of potential targets of Liuwei Dihuang decoction (LWDHD) for the treatment of IVDD. (B) PPI network of significant proteins was extracted from (A) based on BC, CC, DC, EC, LAC, NC. (C) Obtaining I7 core proteins of LWDHD for IVDD were extracted from (B) based on BC, CC, DC, EC, LAC, NC. 
A

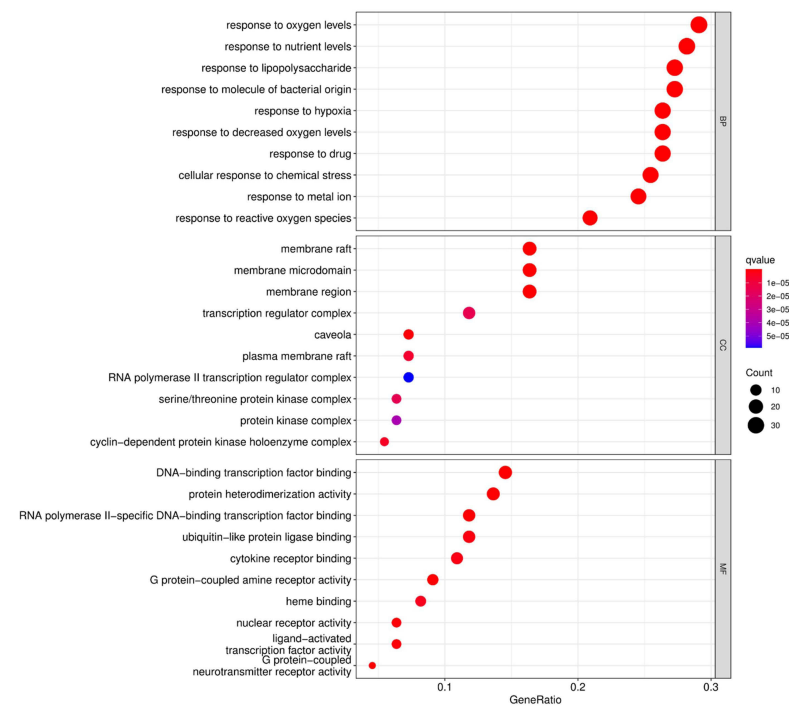

B

C
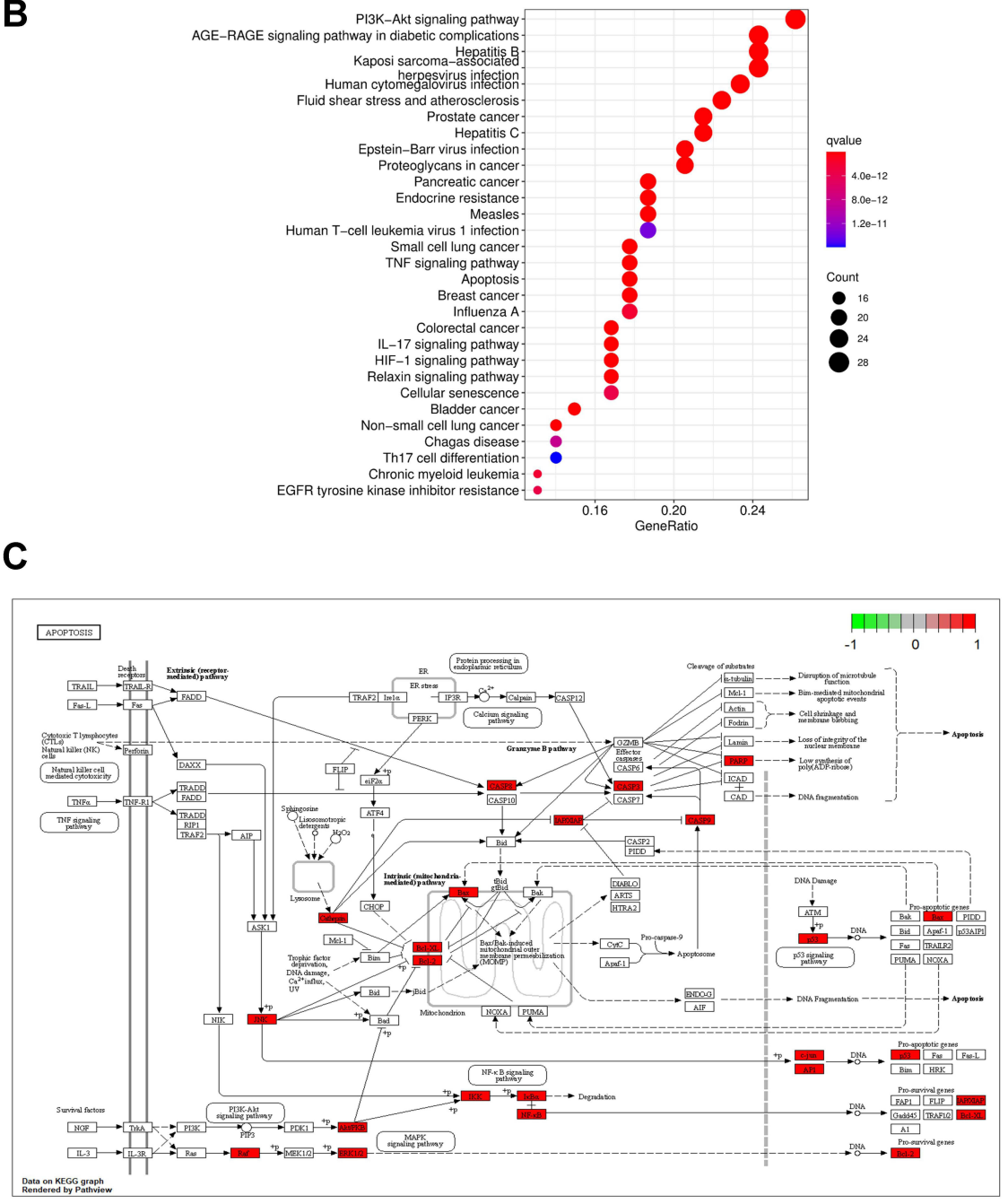

Figure 4 Gene Ontology (GO) and Kyoto Encyclopedia of Genes and Genomes (KEGG) functional analysis. (A) The top 10 of GO enrichment analysis (BP represented biological progress of core targets. CC represented cellular components of core targets. MF represented the molecular function of core targets.) (B) The top 30 signaling pathways from KEGG analysis. (C) The apoptosis signaling pathway was described detailedly. The larger the nodes, the darker the color, and the more genes were enriched. 
signaling pathways closely related to IVDD were listed, including AGE-RAGE signaling pathway, IL-17 signaling pathway, and apoptosis pathway (Figure 4B). Among these enriched pathways, we found that apoptosis played an important role in IVDD, and the predictive targets of apoptosis were shown in detail in Figure 4C.

\section{LWDHD Ameliorates the Progression of Mouse IVDD Model Induced by LSI Surgery}

To further verify the key pharmacological mechanism of LWDHD in the treatment of IVDD as predicted by the network pharmacology analysis in the in vivo animal experiments, IVDD model mice were constructed and administered intragastrically with LWDHD (Figure 5A). The L4-L 5 disc heights of IVDD mice were evaluated by micro-CT analysis at 8-week after LSI surgery. As shown in Figure 5B and $\mathrm{C}$, the disc height of LSI-induced mice was significantly reduced compared with sham mice, and the disc heights of IVDD mice were significantly restored by treatment with LWDHD. Then, the histological changes of L4-L5 IVD were examined by H\&E staining. Consistent with micro-CT results, IVDD mice exhibited ectopic bone formation in cartilaginous endplate (CEP), shrinkage in nucleus pulposus (NP), combined with fissures and folds in annulus fibrosus (AF). The administration of LWDHD significantly reduced the ectopic bone in $\mathrm{CEP}$, and fissures and folds in $\mathrm{AF}$ (Figure 5D and E).

To examine the effects of LWDHD on IVD matrix metabolism, protein levels of Aggrecan, Col2 and corresponding matrix-degrading enzymes Adamts5 and Mmp13 were determined by IHC analysis. As we expected, Aggrecan and Col2 were significantly decreased, and Mmp13 and Adamts5 were dramatically increased in IVDs of IVDD mice. However, LWDHD treatment significantly reversed these alterations in IVDD mice (Figure 5F-J). All these findings suggested that LWDHD administration significantly attenuated the progression of IVDD.

\section{LWDHD Inhibits Apoptosis of IVD Cells in IVDD Mice}

The prediction results of the above network pharmacology analysis showed that LWDHD affected a large number of targets involved in the apoptotic process, including Caspase-3, and P53. Therefore, we further validated the expression changes of these indicators and apoptosisrelated markers (Bcl-2 and Bax) by IHC or IF analysis.
The results of IF analysis showed that Bcl-2 was significantly decreased, whereas the expression of Bax and Caspase-3 were dramatically increased in AF, NP and CEP regions of IVDD mice (Figure 6A-H). LWDHD significantly reversed these alterations of $\mathrm{Bcl}-2$, Bax and Caspase-3. Similarly, the IHC results of P53 also showed that P53 was markedly increased in AF and NP regions of IVDD mice, which was significantly blunted by LWDHD treatment (Figure 6I-K). In addition, the apoptosis of IVD cells was further confirmed via TUNEL staining. We found that the increase in apoptosis rate observed in IVD cells of IVDD mice was largely suppressed by treatment with LWDHD (Figure 6O-P). All these findings indicated that LWDHD inhibited apoptosis of IVD cells and ameliorated IVDD progression by regulating apoptosis-related targets, such as Caspase-3 and P53.

Growing evidence also showed that, in addition to apoptosis, inflammation stimulation contributed to the acceleration of IVDD progression. ${ }^{30} \mathrm{IL}-1 \beta$ is the most important member of the IL-1 family and has a strong pro-inflammatory activity by stimulating the secretion of multiple pro-inflammatory mediators. IL- $1 \beta$ is highly expressed in degenerative intervertebral disk (IVD) tissues and cells, and it has been shown to be involved in multiple pathological processes during disk degeneration, including inflammatory responses, matrix destruction, cellular apoptosis, etc. We examined the expression changes of IL- $1 \beta$ by IHC analysis and the corresponding results showed that IL-1 $\beta$ were markedly increased in AF and NP regions of IVDD mice, which was significantly blunted by LWDHD treatment (Figure 6L-N).

\section{Discussion}

LBP is a clinical common symptom syndrome, characterized by axial pain and restricted movement of the lumbar, ${ }^{31-33}$ which provokes tremendous burdens on the health care system worldwide.$^{34}$ LWDHD, a renowned traditional Chinese herbal compound, has been proven to be therapeutically effective in treating LBP due to its privileged properties. ${ }^{9}$ However, the molecular mechanism of LWDHD on IVDD remained largely elusive, which greatly restricted its modernization and internationalization. ${ }^{13}$ The network pharmacology, an innovative method, systematically detected the mechanisms of the effects of traditional Chinese medicine (TCM) on many types of diseases. ${ }^{14}$ In this study, the effects and underlying pharmacological mechanisms of LWDHD against IVDD were investigated based on network pharmacology analysis coupled with experimental validation. The network analysis identified related potential targets 
A

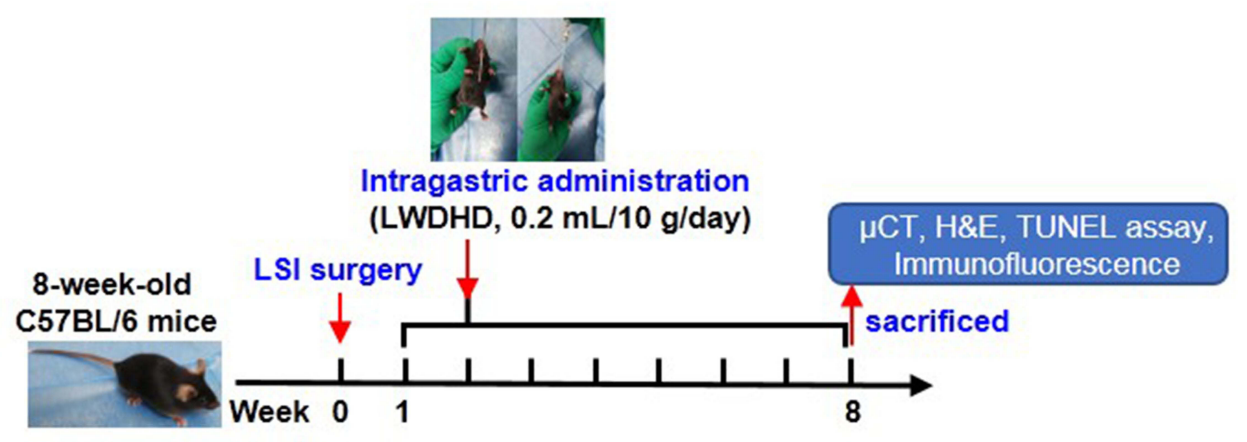

B
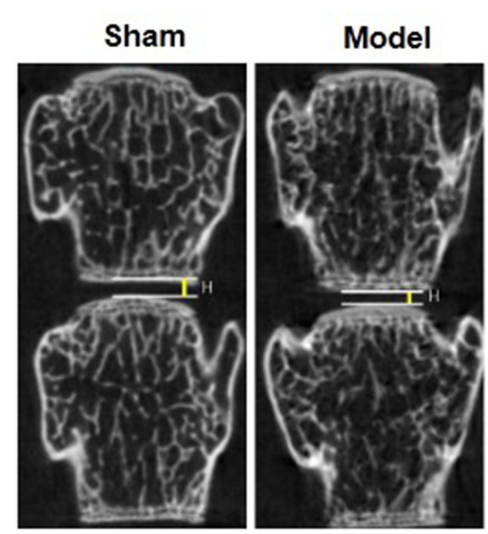

LWDHD

C

E

D

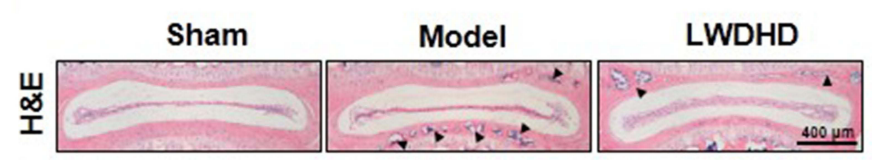

F
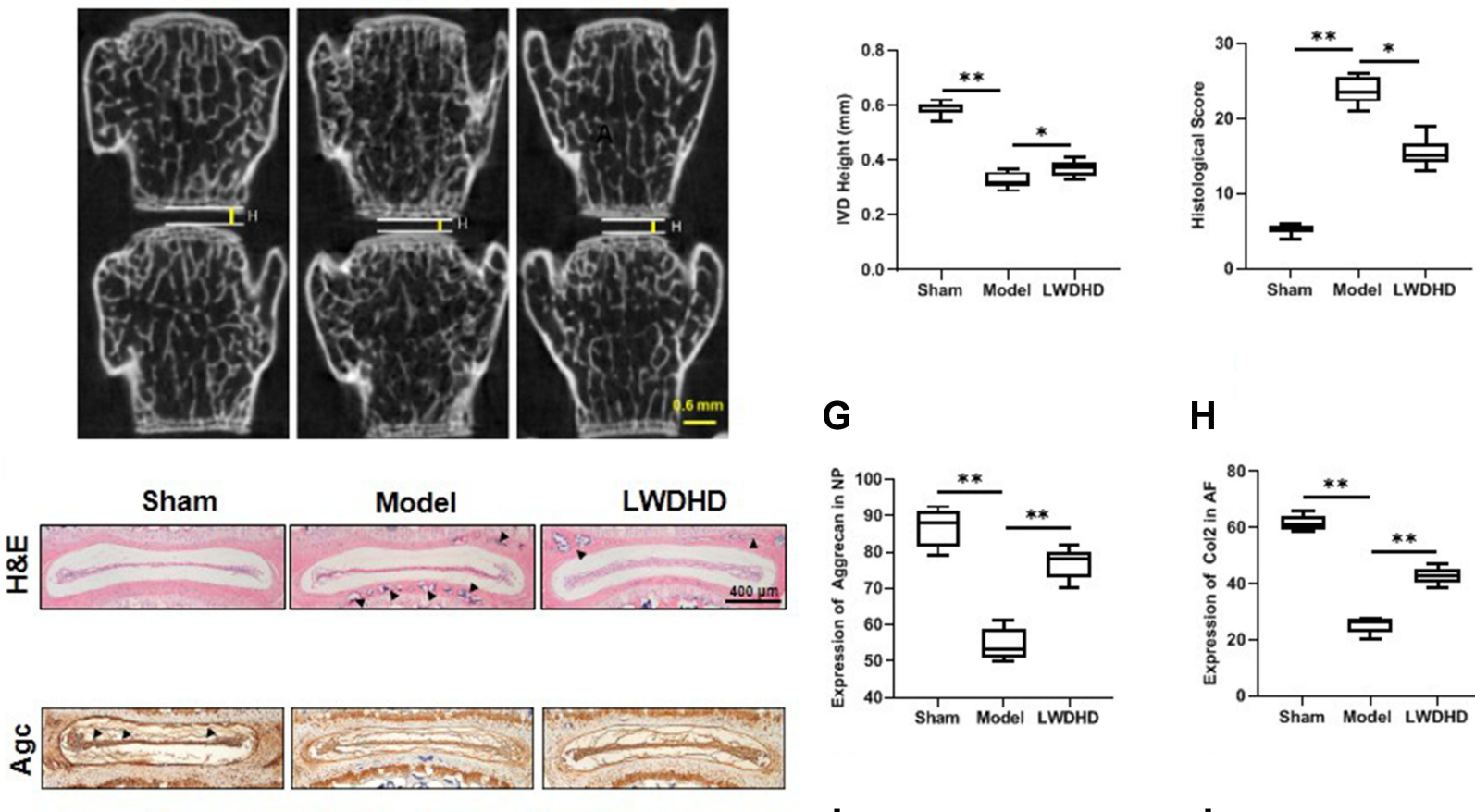

G

H
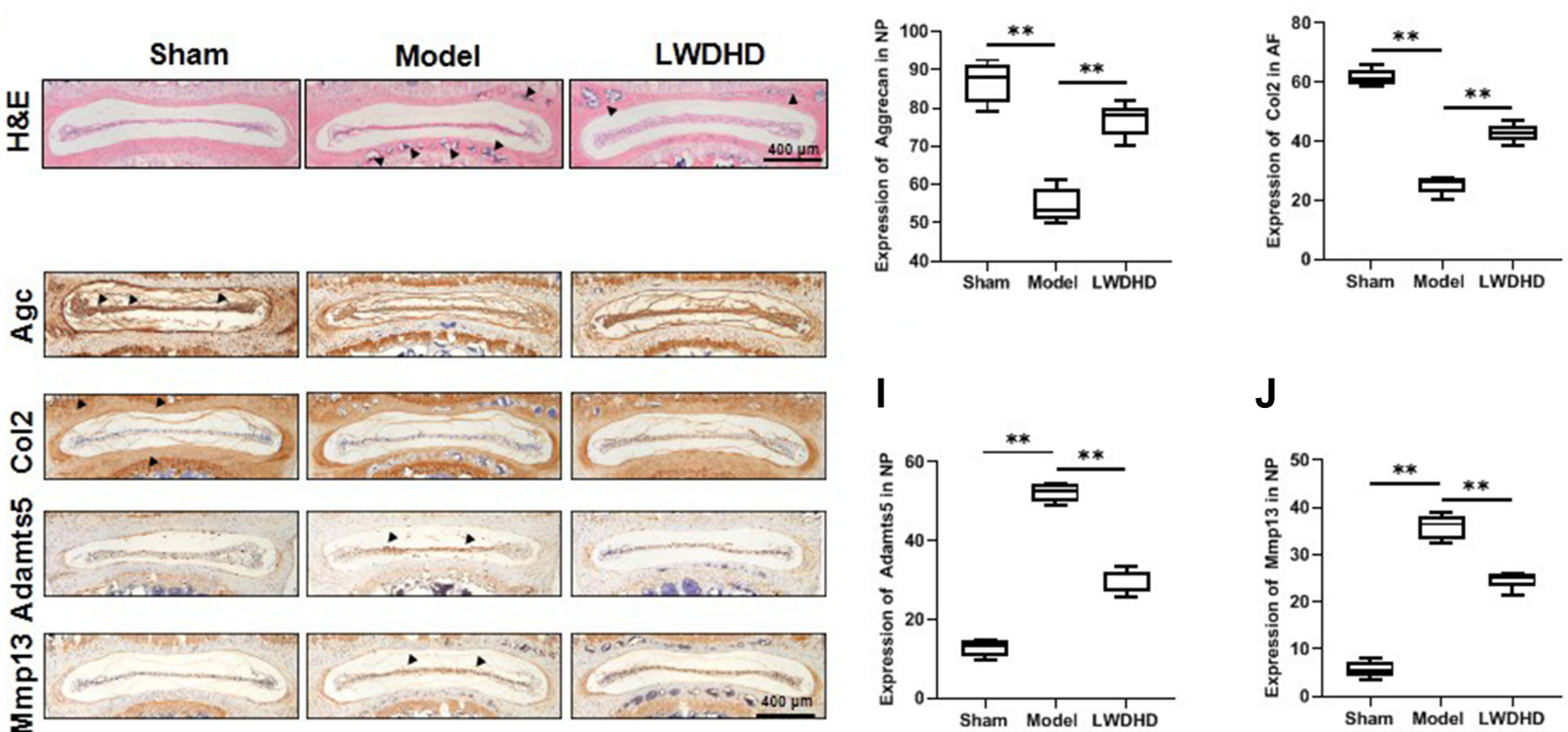

J

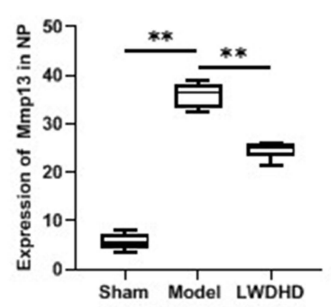

Figure 5 LWDHD attenuated IVDD progression in LSI-induced mouse model. (A) Study design of the project. (B) Evaluation of the height of the IVD by coronal micro-CT of lumbar vertebrae. The yellow line represented the height of the IVD. (C) Quantification of the height of the IVD. (D) Hematoxylin-eosin staining images revealed the morphologic changes of IVD tissues. Black arrows represented ectopic bone formation in CEP. (E) Evaluation of IVDD by histological score. (F) Immunohistochemistry of Aggrecan (Agc), Col2, Mmpl 3 and Adamts5 in IVD. Black arrowheads indicated the high expression of Aggrecan, Col2, Mmp I 3 and Adamts5 in IVD. (G-J) The quantification of Aggrecan, Col2, Mmp 3 and Adamts5 expression. Data were presented as median (min-max) of three independent experiments. $* P<0.05$, $* * P<0.0$ l compared to Sham group.

(Caspase-3, IL-1ß, P53) and specific signaling pathways (particularly the apoptosis signaling). Then, the in vivo mouse IVDD model validation further confirmed that LWDHD inhibited apoptosis and inflammation of IVD tissue and ameliorated the progression of IVDD.
Identification of active ingredients in the formulation provides a new way to explore the mechanisms of treating diseases with TCM. ${ }^{12,35}$ In this study, we found that the top key active ingredients included quercetin, kaempferol, etc. A previous study performed on the rat IVDD model 

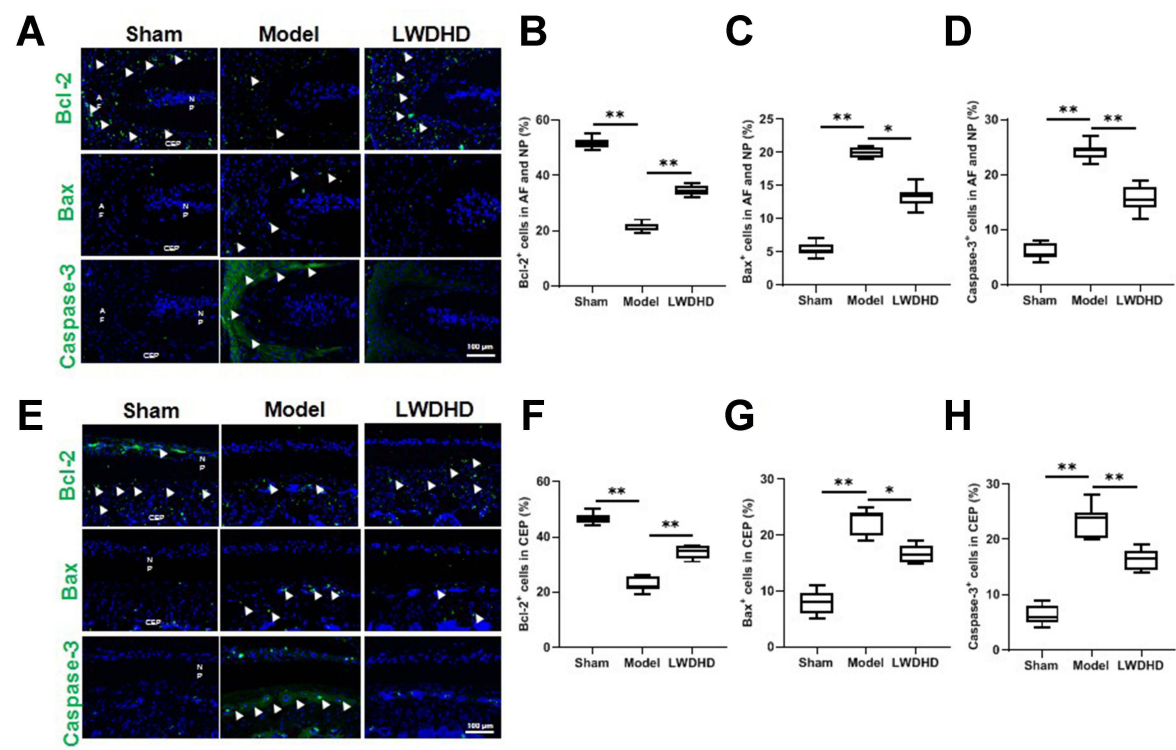

\section{G}

\section{H}
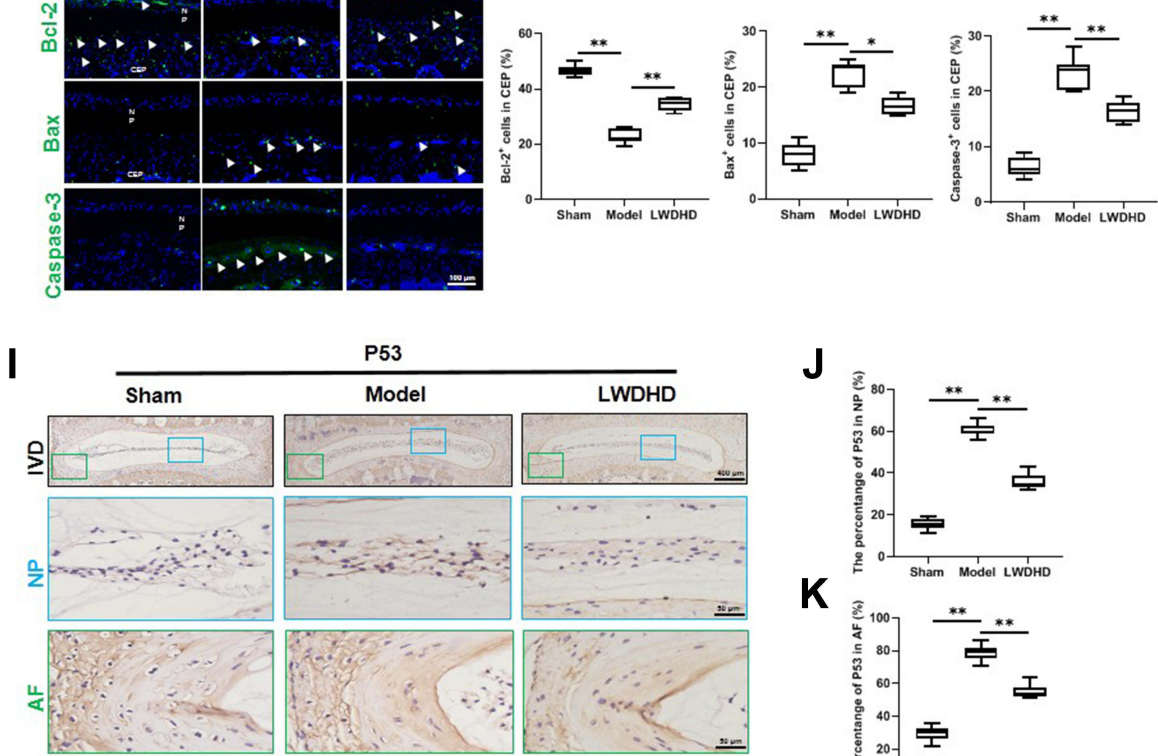

K

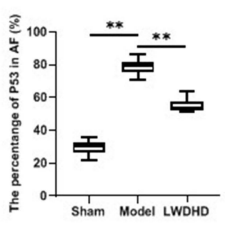

L

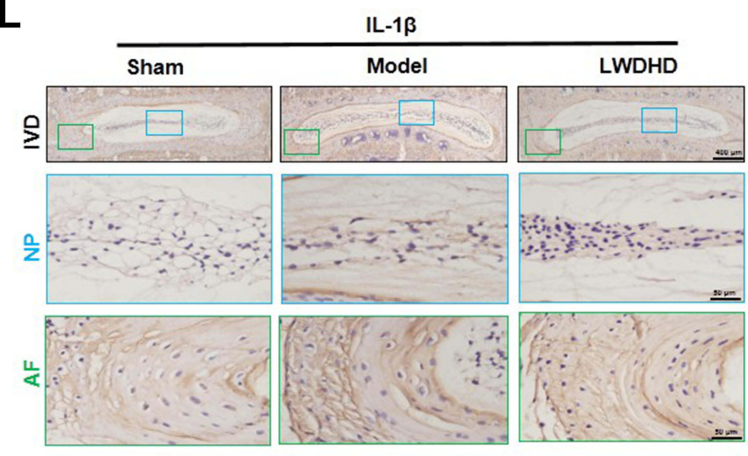

0

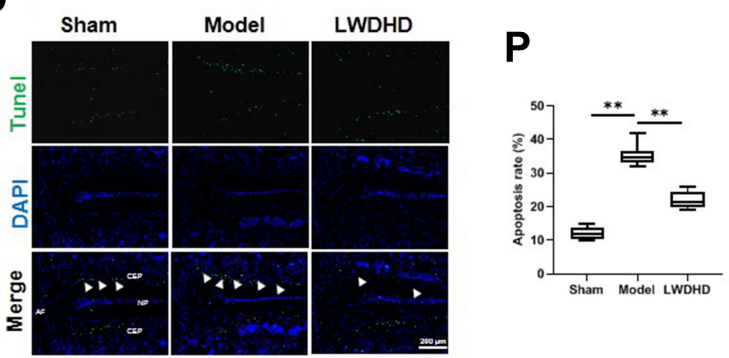

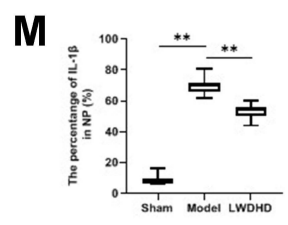

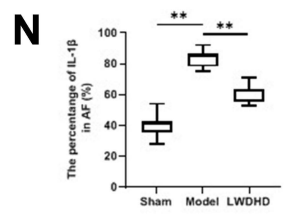

Figure 6 LWDHD inhibited apoptosis of IVD to ameliorate IVDD. (A) Immunofluorescence of Bcl-2, Bax and Caspase-3 in AF and NP. (B-D) The quantification of Bcl-2, Bax and Caspase-3 positive cells in AF and NP. (E) Immunofluorescence of Bcl-2, Bax and Caspase-3 in CEP. (F-H) The quantification of Bcl-2, Bax and Caspase-3 positive cells in CEP. (I) Immunohistochemistry of P53 in NP and AF. ( $\mathbf{J}$ and $\mathbf{K}$ ) The quantification of P53 positive cells in NP and AF. (L) Immunohistochemistry of IL- I $\beta$ in NP and AF. $(\mathbf{M}$ and $\mathbf{N})$ The quantification of IL-I $\beta$ positive cells in NP and AF. (O) Tunel staining was used to detect the apoptosis of IVD. (P) The quantification of TUNEL positive rates in IVD. Data were presented as median (min-max) of three independent experiments. ${ }^{*} P<0.05, * * P<0.01$ compared to Sham group. 
reported that quercetin could alleviate the progression of IVDD. ${ }^{36}$ Recent literature has also documented that kaempferol attenuates IVDD progression due to its unique anti-inflammatory properties. ${ }^{37}$ Thus, all these findings indicated that multiple components in LWDHD played considerable roles in the treatment of IVDD.

To decipher the basic mechanisms of LWDHD against IVDD, the core targets were identified and used for network pharmacological analysis. In the present study, the core targets of active ingredients of LWDHD were identified by the PPI network including Caspase-3, IL-1 $\beta$ and P53, which were closely involved in the apoptosis process. Caspase-3 is the primary effector of apoptosis, and it is also the therapeutic target for regulating IVDD. ${ }^{38}$ Gene silencing of Caspase-3 by siRNA or ablation of Caspase-3 could effectively prevent cell apoptosis and death of IVD, thereby ameliorating IVDD. ${ }^{39}$ Moreover, as an apoptosis marker, elevated P53 stimulates apoptosis of nucleus pulposus cells and accelerates IVDD progression. ${ }^{40}$ Previous clinical research revealed that the level of IL-1 $\beta$ in the IVD of IVDD patients was significantly elevated. ${ }^{41}$ Mechanism study demonstrated that IL-1 $\beta$ accelerated the production of Caspase-3, P53 and Bax in IVD but reduced the production of anti-apoptotic proteins $\mathrm{Bcl}-2$ as well as matrix proteins Col2 and aggrecan. ${ }^{42,43}$ All these findings demonstrated that Caspase-3, P53 and IL$1 \beta$ were of great significance in the development of IVDD.

At present, the pharmacological mechanism of LWDHD against IVDD is still limited. Network target and signaling pathway could perfectly decipher sophisticated interactions between diseases with chemical ingredients in TCM, including LWDHD. KEGG enrichment analysis indicated that the AGE-RAGE signaling pathway, IL-17 signaling pathway, and apoptosis pathway were the potential pathways involved in the intervention of LWDHD for IVDD. Numerous basic studies indicated that apoptosis played a crucial role in the physiological and pathological processes of IVD. ${ }^{44,45}$ Exceeding apoptosis decreased the production of extracellular matrix in IVD and accelerated the pathogenesis of IVDD. ${ }^{46}$ In vivo and in vitro evidence suggested that inhibition of apoptosis was an efficient strategy to prevent the death of IVD cells and ameliorate the progression of IVDD. ${ }^{47,48}$ Consistent with these findings, evidence from our in vivo animal studies showed that LWDHD significantly reversed the increased expression of Bax, Caspase-3, P53 and IL-1 $\beta$, as well as the increased TUNEL positive rate in IVD tissues of IVDD mice. All these findings uncovered the possible mechanisms of therapeutic effects of LWDHD on IVDD or LBP are to regulate the aberrant expression of apoptosis-related proteins and reduce increased DNA damage, thereby weakening the apoptosis of IVD tissue to ameliorate the progression of IVDD. LWDHD could treat IVDD via 'multi-ingredients, multi-target, multipathway mode.

However, there were some limitations in the study. First, we paid more attention to the components and targets at the top of the list, while ignoring the components and targets at the back of the list, which may lead to a slight deviation of study results. Secondly, experimental validation of predicted targets and pathways was relatively limited. We chose to verify only the crucial role of the apoptosis pathway in the treatment of IVDD with LWDHD, while other predicted important targets and pathways require further experimental verification in the future.

\section{Conclusion}

In summary, to explore the pharmacological mechanisms of LWDHD against IVDD, network pharmacology analysis and in vivo experimental validations were carried out. The network pharmacological analysis predicted that LWDHD exerted its therapeutic effects against IVDD via regulating multiple ingredients, targets and pathways. The animal experiments confirmed that the mechanism of LWDHD treating IVDD was to inhibit the apoptosis of IVD cells through regulation of Caspase-3, P53 and IL-1 $\beta$, thereby improving the progression of IVDD. Taken together, this study provides an optimized approach for elucidating the pharmacological mechanisms of LWDHD and supplying novel targets for the treatment of IVDD.

\section{Acknowledgments}

This study was financially supported by National Natural Science Foundation of China (No.: 81804121, 82174140, 82174401, 82104164, 81973870, 81973881, 81904053), Postdoctoral Research Foundation of China (No.: 2018M632154), Natural Science Foundation of Zhejiang Province (No.: LY19H270006 and LQ19H080001), Traditional Chinese Medical Administration of Zhejiang Province (No.: 2022ZX005, 2022ZB119, 2021ZB090), Zhejiang Medical and Health Science and Technology Project (No.: 2021KY222), Research Project of Zhejiang Chinese Medical University (No.: 2021JKZDZC02, 2021JKZKTS036A， 2021JKJNTZ022B， 2019ZG25, 
2018ZR06), National Undergraduate Innovation and Entrepreneurship Training Program (No.: 202110344005, 202110344025, S202110344007, 202010344004), General Research Project of Zhejiang Provincial Education Department "Special Project for the Reform of Cultivation Mode of Professional Degree Graduate Students in Higher Education Institutions" (No.: Y202145932), Postgraduate Science Research Fund of Zhejiang Chinese Medical University (No.: 2021YKJ02, 2020YKJ07).

\section{Disclosure}

The authors declare that they have no competing interests.

\section{References}

1. Chenot JF, Greitemann B, Kladny B, Petzke F, Pfingsten M, Schorr SG. Non-specific low back pain. Dtsch Arztebl Int. 2017;114 (51-52):883-890. doi:10.3238/arztebl.2017.0883

2. Van Hoof W, O'Sullivan K, O'Keeffe M, Verschueren S, O'Sullivan P, Dankaerts W. The efficacy of interventions for low back pain in nurses: a systematic review. Int $J$ Nurs Stud. 2018;77:222-231. doi:10.1016/j.ijnurstu.2017.10.015

3. Molladavoodi S, McMorran J, Gregory D. Mechanobiology of annulus fibrosus and nucleus pulposus cells in intervertebral discs. Cell Tissue Res. 2020;379(3):429-444. doi:10.1007/s00441-019-03136-1

4. Sun Z, Wang HQ, Liu ZH, et al. Down-regulated CK8 expression in human intervertebral disc degeneration. Int J Med Sci. 2013;10 (8):948-956. doi:10.7150/ijms.5642

5. Taylor S, Bishop A. Patient and public beliefs about the role of imaging in the management of non-specific low back pain: a scoping review. Physiotherapy. 2020;107:224-233. doi:10.1016/j. physio.2019.08.014

6. Cazzanelli P, Wuertz-Kozak K. MicroRNAs in intervertebral disc degeneration, apoptosis, inflammation, and mechanobiology. Int J Mol Sci. 2020;21:10. doi:10.3390/ijms21103601

7. Lyu FJ, Cui H, Pan H, et al. Painful intervertebral disc degeneration and inflammation: from laboratory evidence to clinical interventions. Bone Res. 2021;9(1):7. doi:10.1038/s41413-020-00125-x

8. Castillo ER, Lieberman DE. Lower back pain. Evol Med Public Health. 2015;2015(1):2-3. doi:10.1093/emph/eou034

9. Luo Y, Huang J, Xu L, Zhao W, Hao J, Hu Z. Efficacy of Chinese herbal medicine for lumbar disc herniation: a systematic review of randomized controlled trials. J Tradit Chin Med. 2013;33 (6):721-726. doi:10.1016/S0254-6272(14)60003-0

10. Ge JR, Xie LH, Chen J, et al. Liuwei Dihuang Pill () Treats Postmenopausal Osteoporosis with Shen (Kidney) Yin Deficiency via Janus Kinase/Signal transducer and activator of transcription signal pathway by up-regulating cardiotrophin-like Cytokine Factor 1 expression. Chin J Integr Med. 2018;24(6):415-422. doi:10.1007/ s11655-016-2744-2

11. Zeng J, Zhang X, Wang J, Cheng X, Zhang Y, Zhou W. Comparison of donepezil, memantine, melatonin, and liuwei dihuang decoction on behavioral and immune endocrine responses of aged senescence-accelerated mouse resistant 1 mice. Front Pharmacol. 2020;11:350. doi:10.3389/fphar.2020.00350

12. He D, Huang JH, Zhang ZY, et al. A network pharmacology-based strategy for predicting active ingredients and potential targets of LiuWei DiHuang pill in treating Type 2 diabetes mellitus. Drug Des Devel Ther. 2019;13:3989-4005. doi:10.2147/DDDT.S216644
13. An L, Lin Y, Li L, et al. Integrating network pharmacology and experimental validation to investigate the effects and mechanism of astragalus flavonoids against hepatic fibrosis. Front Pharmacol. 2020;11:618262. doi:10.3389/fphar.2020.618262

14. Shi H, Dong C, Wang M, et al. Exploring the mechanism of Yizhi Tongmai decoction in the treatment of vascular dementia through network pharmacology and molecular docking. Ann Transl Med. 2021;9(2):164. doi:10.21037/atm-20-8165

15. Zhao L, Guo R, Cao N, et al. An integrative pharmacology-based pattern to uncover the pharmacological mechanism of ginsenoside $\mathrm{H}$ dripping pills in the treatment of depression. Front Pharmacol. 2020;11:590457. doi:10.3389/fphar.2020.590457

16. Guo C, Kang X, Cao F, et al. Network pharmacology and molecular docking on the molecular mechanism of Luo-hua-zi-zhu (LHZZ) granule in the prevention and treatment of bowel precancerous lesions. Front Pharmacol. 2021;12:629021. doi:10.3389/ fphar.2021.629021

17. Liu J, Liu J, Tong X, et al. Network pharmacology prediction and molecular docking-based strategy to discover the potential pharmacological mechanism of Huai Hua San against ulcerative colitis. Drug Des Devel Ther. 2021;15:3255-3276. doi:10.2147/DDDT.S319786

18. Xia H, Liu J, Yang W, et al. Integrated strategy of network pharmacological prediction and experimental validation elucidate possible mechanism of $\mathrm{Bu}$-Yang herbs in treating postmenopausal osteoporosis via ESR1. Front Pharmacol. 2021;12:654714. doi:10.3389/ fphar.2021.654714

19. Ru J, Li P, Wang J, et al. TCMSP: a database of systems pharmacology for drug discovery from herbal medicines. $J$ Cheminform. 2014;6:13. doi:10.1186/1758-2946-6-13

20. Feng W, Ao H, Yue S, Peng C. Systems pharmacology reveals the unique mechanism features of Shenzhu Capsule for treatment of ulcerative colitis in comparison with synthetic drugs. Sci Rep. 2018;8(1):16160. doi:10.1038/s41598-018-34509-1

21. UniProt C. UniProt: a worldwide hub of protein knowledge. Nucleic Acids Res. 2019;47(D1):D506-D515.

22. Stelzer G, Dalah I, Stein TI, et al. In-silico human genomics with GeneCards. Hum Genomics. 2011;5(6):709-717. doi:10.1186/14797364-5-6-709

23. Amberger JS, Bocchini CA, Schiettecatte F, Scott AF, Hamosh A. OMIM.org: online Mendelian Inheritance in Man (OMIM(R)), an online catalog of human genes and genetic disorders. Nucleic Acids Res. 2015;43(Databaseissue):D789-798. doi:10.1093/nar/ gku1205

24. Wishart DS, Feunang YD, Guo AC, et al. DrugBank 5.0: a major update to the DrugBank database for 2018. Nucleic Acids Res. 2018;46(D1):D1074-D1082. doi:10.1093/nar/gkx1037

25. Barbarino JM, Whirl-Carrillo M, Altman RB, Klein TE. PharmGKB: a worldwide resource for pharmacogenomic information. Wiley Interdiscip Rev Syst Biol Med. 2018;10(4):e1417. doi:10.1002/ wsbm. 1417

26. Szklarczyk D, Morris JH, Cook H, et al. The STRING database in 2017: quality-controlled protein-protein association networks, made broadly accessible. Nucleic Acids Res. 2017;45(D1):D362-D368. doi:10.1093/nar/gkw937

27. Li Q, Hu S, Huang L, Zhang J, Cao G. Evaluating the therapeutic mechanisms of selected active compounds in Cornus officinalis and Paeonia lactiflora in rheumatoid arthritis via network pharmacology analysis. Front Pharmacol. 2021;12:648037. doi:10.3389/ fphar.2021.648037

28. Fu F, Bao R, Yao S, et al. Aberrant spinal mechanical loading stress triggers intervertebral disc degeneration by inducing pyroptosis and nerve ingrowth. Sci Rep. 2021;11(1):772. doi:10.1038/s41598-02080756-6 
29. Yu H, Yao S, Zhou C, et al. Morroniside attenuates apoptosis and pyroptosis of chondrocytes and ameliorates osteoarthritic development by inhibiting NF-kappaB signaling. $J$ Ethnopharmacol. 2021;266:113447. doi:10.1016/j.jep.2020.113447

30. Patil P, Niedernhofer LJ, Robbins PD, Lee J, Sowa G, Vo N. Cellular senescence in intervertebral disc aging and degeneration. Curr Mol Biol Rep. 2018;4(4):180-190. doi:10.1007/s40610-018-0108-8

31. Driscoll T, Jacklyn G, Orchard J, et al. The global burden of occupationally related low back pain: estimates from the Global Burden of Disease 2010 study. Ann Rheum Dis. 2014;73(6):975-981. doi:10.1136/annrheumdis-2013-204631

32. Foster NE, Anema JR, Cherkin D, et al. Prevention and treatment of low back pain: evidence, challenges, and promising directions. The Lancet. 2018;391(10137):2368-2383. doi:10.1016/S0140-6736(18)30489-6

33. Yang G, Wang Y, Zeng Y, et al. Rapid health transition in China, 1990-2010: findings from the Global Burden of Disease Study 2010. The Lancet. 2013;381(9882):1987-2015. doi:10.1016/S01406736(13)61097-1

34. Ge J, Cheng X, Yan Q, et al. Calcitonin inhibits intervertebral disc degeneration by regulating protein kinase C. J Cell Mol Med. 2020;24(15):8650-8661. doi:10.1111/jcmm.15496

35. Li S, Zhang B. Traditional Chinese medicine network pharmacology: theory, methodology and application. Chin J Nat Med. 2013;11 (2):110-120. doi:10.1016/S1875-5364(13)60037-0

36. Wang D, He X, Wang D, et al. Quercetin suppresses apoptosis and attenuates intervertebral disc degeneration via the SIRT1-autophagy pathway. Front Cell Dev Biol. 2020;8:613006. doi:10.3389/ fcell.2020.613006

37. Ren J, Lu Y, Qian Y, Chen B, Wu T, Ji G. Recent progress regarding kaempferol for the treatment of various diseases. Exp Ther Med. 2019;18(4):2759-2776. doi:10.3892/etm.2019.7886

38. Sudo H, Minami A. Caspase 3 as a therapeutic target for regulation of intervertebral disc degeneration in rabbits. Arthritis Rheum. 2011;63 (6):1648-1657. doi:10.1002/art.30251

39. Ohnishi T, Yamada K, Iwasaki K, et al. Caspase-3 knockout inhibits intervertebral disc degeneration related to injury but accelerates degeneration related to aging. Sci Rep. 2019;9(1):19324. doi:10.1038/s41598-019-55709-3
40. Zhang K, Zhang Y, Zhang C, Zhu L. Upregulation of P53 promotes nucleus pulposus cell apoptosis in intervertebral disc degeneration through upregulating NDRG2. Cell Biol Int. 2021;45(9):1966-1975. doi:10.1002/cbin.11650

41. Kepler CK, Markova DZ, Dibra F, et al. Expression and relationship of proinflammatory chemokine RANTES/CCL5 and cytokine IL-1 $\beta$ in painful human intervertebral discs. Spine. 2013;38(11):873-880. doi:10.1097/BRS.0b013e318285ae08

42. Wang $K$, Chen T, Ying X, et al. Ligustilide alleviated IL-1 $\beta$ induced apoptosis and extracellular matrix degradation of nucleus pulposus cells and attenuates intervertebral disc degeneration in vivo. Int Immunopharmacol. 2019;69:398-407. doi:10.1016/j.intimp.201 9.01.004

43. Jiang Y, Xie Z, Yu J, Fu L. Resveratrol inhibits IL-1 $\beta$-mediated nucleus pulposus cell apoptosis through regulating the PI3K/Akt pathway. Biosci Rep. 2019;39:3. doi:10.1042/BSR20190043

44. Long J, Wang X, Du X, et al. JAG2/Notch2 inhibits intervertebral disc degeneration by modulating cell proliferation, apoptosis, and extracellular matrix. Arthritis Res Ther. 2019;21(1):213. doi:10.1186/s13075-019-1990-z

45. Majtnerova P, Rousar T. An overview of apoptosis assays detecting DNA fragmentation. Mol Biol Rep. 2018;45(5):1469-1478. doi: 10.1007/s11033-018-4258-9

46. Sudo H, Minami A. Caspase 3 as a therapeutic target for regulation of intervertebral disc degeneration in rabbits. Arthritis Rheum-Us. 2011;63(6):1648-1657.

47. Hu B, Zhang S, Liu W, et al. Inhibiting heat shock protein 90 protects nucleus pulposus-derived stem/progenitor cells from compression-induced necroptosis and apoptosis. Front Cell Devel Biol. 2020;8:685. doi:10.3389/fcell.2020.00685

48. Xie L, Huang W, Fang Z, et al. CircERCC2 ameliorated intervertebral disc degeneration by regulating mitophagy and apoptosis through miR-182-5p/SIRT1 axis. Cell Death Dis. 2019;10(10):751. doi:10.1038/s41419-019-1978-2

\section{Publish your work in this journal}

Drug Design, Development and Therapy is an international, peerreviewed open-access journal that spans the spectrum of drug design and development through to clinical applications. Clinical outcomes, patient safety, and programs for the development and effective, safe, and sustained use of medicines are a feature of the journal, which has also been accepted for indexing on PubMed Central. The manuscript management system is completely online and includes a very quick and fair peer-review system, which is all easy to use. Visit http://www. dovepress.com/testimonials.php to read real quotes from published authors. 